\title{
Gerencia del conocimiento en empresas cerveceras. Estudio de un caso
}

\author{
Batlle Rois-Méndez, Francisco* \\ Inciarte Osorio, Bárbara**
}

\begin{abstract}
Resumen
La investigación se realizó con el propósito de determinar el desarrollo de la gerencia del conocimiento en empresas cerveceras del Municipio San Francisco del estado Zulia, Venezuela. La investigación fue descriptiva; con un diseño de campo, no experimental transversal. La muestra estuvo constituida por 98 sujetos integrados por los gerentes, superintendentes y empleados de Cervecería Polar C.A., Planta Modelo. Resultados indican que la generación del conocimiento, la dimensión tecnológica, la dimensión cultural y el liderazgo son los principales elementos de la gerencia del conocimiento desarrollada en este sector empresarial; destacándose como conclusión que el desarrollo de los aspectos tecnológicos como la estrategia es lo que le ha permitido mantenerse como líder en el mercado gracias a las innovaciones, a la tecnología de punta que aplica a los procesos productivos y a un liderazgo dirigido a fomentar el trabajo en equipo como valor organizacional.
\end{abstract}

Palabras clave: Gerencia del conocimiento, innovación, creatividad, comunidades de aprendizaje, liderazgo.

* Licenciado en Educación (UNICA, 1998). Asesor Metodológico y Auxiliar de Investigación (LUZ-CEE, 1999). Magíster Scientiarum en Docencia para Educación Superior (UNERMB, 2004). Doctor en Ciencias Gerenciales (URBE, 2007). Coordinador del Programa de Postgrado en Educación Avanzada Continua (URBE). PPI: 11-349. Móvil: 0414-9665766 / 0416 6605492. E-mail: francisco.batlle@urbe.edu/ franbatlle@cantv.net

** Licenciada en Contaduría Pública (LUZ, 1987). Magíster Scientiarum en Gerencia Empresarial (URBE, 2003). Jefe de Contabilidad y Costo (Cervecería Polar C.A. - Planta Modelo). Maracaibo - Venezuela. Movil: 0414-6342340. E-mail: barbara.inciarte@empresas-polar.com 


\title{
Knowledge Management in Beer Companies. A Case Study
}

\begin{abstract}
The study was made in order to determine the development of knowledge management in brewery enterprises of the San Francisco Municipality, State of Zulia, Venezuela. The research was descriptive; with a non-experimental, transversal field design. The sample consisted of 98 subjects, including managers, superintendents and employees at Cervecería Polar C.A., Planta Modelo. Conclusions were that the generation of knowledge, the technological dimension, the cultural dimension and leadership are key elements of knowledge management developed in this industry, highlighting the development of technological aspects as the strategy that has allowed it to remain the leader in the market, thanks to innovations in cutting-edge technology applied to production processes and a leadership that encourages teamwork as an organizational value.
\end{abstract}

Key words: Knowledge management, innovation, creativity, learning communities, leadership.

\section{Introducción}

Los cambios que se suscitaron para finales del siglo $\mathrm{XX}$ y se siguen produciendo, no tienen precedente histórico; son transformaciones profundas, permanentemente aceleradas e interdependientes en todos los ámbitos: social, político, científico, industrial y tecnológico. El paso del cambio tecnológico y social continúa día a día esta tendencia, forzando los límites del conocimiento, así como su desarrollo, relacionabilidad y visión de futuro.

Es así como, según Ferrer (2001), afloran los conceptos de conocimiento y sabiduría, presentándolos como opciones favorecedoras de la innovación que se hace necesaria en las empresas ante la presión de las realidades emergentes, consecuencia de los vertiginosos cambios del mundo. Agrega el autor citado que el conocimiento es organización y asimilación de los datos, mientras que la sabiduría es la aptitud y la disposición de generar nuevas informaciones y de desa- rrollar nuevos conocimientos a partir de los datos asimilados en función del mejoramiento específico de un sujeto, de una organización o de una determinada realidad.

Sobre la base de los planteamientos anteriores, se puede inferir que el problema del conocimiento que se presenta en la actualidad, deriva de la aptitud y la disposición, no sólo del individuo sino también de las organizaciones, para fomentar y desarrollar procesos tendientes a la generación de conocimiento pertinente y contextualizado.

En este sentido, Nonaka (2000: 24) afirma que "en una economía cuya única certidumbre es la incertidumbre, la mejor fuente para obtener ventajas competitivas duraderas es el conocimient". El trabajador de organizaciones que emplean la gerencia del conocimiento debe mantenerse actualizado y crear sentido de pertinencia, lo que le permite formar parte de comunidades internacionales de conocimiento.

En Venezuela no son muchas las organizaciones que están totalmente in- 
Gerencia del conocimiento en empresas cerveceras

Batlle Rois-Méndez, Francisco y Inciarte Osorio, Bárbara

volucradas en esta forma innovadora de gerenciar, sólo las más avanzadas, como Petróleos de Venezuela que le dio especial importancia a los Centros de Excelencia, a las comunidades de conocimientos y al desarrollo de competencias técnicas, entre otras que demuestran la importancia que le han dado al conocimiento como una forma de mantener la competitividad en el negocio petrolero.

En este sentido, Ferrer (2001) señala que es urgente reconocer en el país estas tendencias vigentes en el mundo contemporáneo, haciéndose necesario utilizar la información para transformarla en conocimiento y, al mismo tiempo, en beneficios competitivos para las organizaciones venezolanas, convirtiéndose en una salida pertinente para la crisis que actualmente atraviesa el país.

En este orden de ideas, se presentan los procesos generadores de conocimiento como la vía idónea para establecer estas nuevas formas de hacer las cosas, para alcanzar un país moderno acorde con los tiempos actuales. Las instituciones educativas, los centros de investigación, consultoría e innovación y otros relacionados con la construcción de aprendizajes, serán los encargados de optimizar y operativizar la práctica de la gerencia del conocimiento como mecanismo para lograr los mejores resultados para el país.

Por su parte, las empresas cerveceras que funcionan en Venezuela han realizado procesos de benchmarking para identificar las mejores prácticas gerenciales que les permitan ubicarse como líderes en un negocio significativamente competitivo. Estas empresas han evolucionado en los últimos años hacia una ge- rencia del conocimiento orientada a fomentar la innovación, para lo cual se ha hecho imprescindible la participación de los trabajadores y contar con el apoyo de herramientas electrónicas que faciliten la organización, acopio, ordenamiento, clasificación, redistribución y administración del conocimiento.

Como factores considerados por la empresa para el éxito del sistema se presentan: la disposición a compartir información, la integración del equipo de recursos humanos y la generación de una cultura hacia el conocimiento. Este último elemento es quizás el reto más grande que afrontaría la organización para estimular la innovación, la creatividad, el intercambio de conocimiento y experiencias, lo cual debe estar fuertemente apoyado en un sistema de reconocimientos orientado a fortalecer actitudes y conductas de los trabajadores, hacia la gestión de su conocimiento.

En pocas palabras, la gestión del conocimiento es efectiva en tanto que permite documentar la generación de valor que cada trabajador aporta al negocio. Se podría prever que en el futuro los reconocimientos estarán basados en la cantidad de conocimiento que genere el trabajador para dar valor agregado a la organización y que este conocimiento se traduzca en un incremento de los estándares de calidad que coadyuven en la satisfacción de los clientes y consumidores de los productos que oferta la organización.

Es así como los procesos inherentes a la gerencia del conocimiento se han consolidado con la implantación de Sistemas de Gestión del Conocimiento y con Modelos Corporativos de Gestión de la Gente, sin existir hasta ahora, un estudio 
que analice su desarrollo y caracterice el proceso de gerencia del conocimiento en su ámbito particular.

Siendo el análisis de la gerencia del conocimiento una necesidad sentida en el ámbito de la gerencia de empresas, se planteó como objetivo determinar el desarrollo de la gerencia del conocimiento en empresas cerveceras, el cual se logró analizando cuatro elementos constitutivos de la misma: a) el proceso de generación del conocimiento desarrollado en empresas cerveceras; b) la dimensión tecnológica con la cual se administra la gerencia del conocimiento en estas organizaciones; c) la dimensión cultural sobre la cual se soporta la gerencia del conocimiento; y d) el liderazgo ejercido por los gerentes del conocimiento en este sector empresarial.

En atención a estos planteamientos, se diseñó una investigación descriptiva, de campo, no experimental; en la cual la información necesaria fue recogida de las fuentes primarias a través de un cuestionario aplicado a 98 sujetos: 19 gerentes y superintendentes y 79 empleados de Cervecería Polar C.A., Planta Modelo, empresa cervecera de clase mundial, ubicada en el Municipio San Francisco del Estado Zulia, Venezuela.

\section{Gerencia del conocimiento. Breve enfoque teórico}

La gerencia del conocimiento es definida por Udaondo (2000) como el esfuerzo de una organización por conseguir, organizar, distribuir y compartir los conocimientos entre todos los empleados. Por su parte, Pavez (2000), conside- ra que la gerencia del conocimiento debe ser entendida como la instancia de gestión mediante la cual se obtiene, despliega o utiliza una variedad de recursos básicos para apoyar el desarrollo del conocimiento dentro de la organización.

Es importante destacar que la definición de Pavez (2000) se ajustó a los requerimientos de la investigación por cuanto considera la gerencia del conocimiento como una parte de la gestión organizacional de una empresa, tal como se maneja de forma operativa en las empresas cerveceras.

Por otra parte, dentro de las teorías que fundamentan la gerencia del conocimiento y los procesos que ella conlleva, se analizaron los postulados de Nonaka y Takeuchi (2000), Argyris (2000) y Garvin (2000); por considerar que generan aportes significativos a la investigación ya que se alinean con los procesos desarrollados en las empresas cerveceras.

De esta forma, la Empresa creadora de Conocimiento, teoría formulada por Nonaka y Takeuchi (2000), se fundamenta en el hecho de que en una economía cuya única certidumbre es la incertidumbre, la mejor fuente para obtener ventajas competitivas duraderas es el conocimiento. Cuando de la noche a la mañana cambian los mercados, proliferan las tecnologías, se multiplican los competidores, y los productos quedan obsoletos, solo alcanzaran el éxito las empresas que de un modo consistente creen nuevo conocimiento, lo difundan por todas las empresas y lo incorporen rápidamente a la nueva tecnologías y productos. Esas son las actividades que definen a la empresa creadora de cono- 
Gerencia del conocimiento en empresas cerveceras

Batlle Rois-Méndez, Francisco y Inciarte Osorio, Bárbara

cimiento, donde todo el negocio gira sobre la innovación continuada.

Por su parte, Un Enfoque Clave para el Aprendizaje de los Directivos, es la teoría propuesta por Argyris (2000), y señala que toda empresa que aspira triunfar en el ambiente competitivo debe empezar por resolver un dilema fundamental: el éxito en el mercado depende cada vez más del aprendizaje.

La mayoría de las empresas no sólo tienen una tremenda dificultad en resolver este dilema del aprendizaje, sino que ni siquiera son conscientes de su existencia. El motivo: entienden mal lo qué es el aprendizaje y cómo originarlo. Como consecuencia, tienden a cometer equivocaciones en sus intentos de convertirse en una organización con capacidad para aprender.

La mayoría de las personas definen el aprendizaje de un modo demasiado estrecho, como una simple resolución de problemas, por lo que centran su atención en identificar y corregir errores en el entorno que les rodea. La resolución de problemas es importante; pero, para que el aprendizaje persista, los directivos y los empleados deben mirar hacia dentro. Necesitan considerar críticamente su propio comportamiento, determinar los modos con los que a menudo, inadvertidamente, contribuyen a crear problemas a la empresa y luego cambian su modo de actuar. En particular, deben aprender cómo, el mismo modo de definir y resolver los problemas, puede ser una fuente de problemas en sí mismo.

En otro orden de ideas, Garvin (2000) considera que las empresas intentan perfeccionarse y obtener ventajas competitivas, por eso proliferan los pro- gramas de mejora continua. Desafortunadamente, los programa que no dan ningún resultado superan a los exitosos, y el porcentaje de mejora sigue a nivel muy bajo. Eso se debe que la mayoría de las empresas no han captado una verdad básica. Para que las personas y las empresas puedan mejorar, antes tienen que aprender. Y para hacerlo deben mirar más allá de la retórica y la alta filosofía, y centrarse en los fundamentos.

Antes que la empresa pueda convertirse en una auténtica organización que aprende, debe resolver tres cuestiones fundamentales. La primera es una cuestión de significado: una definición de organizaciones que aprenden bien fundada y de fácil aplicación. La segunda es de gestión: establecer unas orientaciones operativas más clara para la práctica. Por último, mejores sistemas de evaluación con los cuales estimar el nivel y el ritmo de aprendizaje de la empresa. Empleando estos tres elementos como marco general, Garvin (2000) define a la organización que aprende como experta en cinco actividades principales a saber: resolución sistemática de los problemas, experimentación de nuevos enfoques, aprender de las experiencias del pasado, aprender de los demás y transmisión del conocimiento.

\section{Generación de conocimiento}

Las organizaciones saludables generan y usan conocimiento. A medida que las organizaciones interactúan con sus entornos, absorben información, la convierten en conocimiento y llevan a cabo acciones sobre la base de la combinación de ese conocimiento y de sus ex- 
periencias, valores y normas internas. En este sentido, Davenport y Prusak (2001: 61) afirman que "muchas empresas abordan la generación de conocimiento como una "caja negra", y, fundamentalmente, sólo intentan contratar personas inteligentes y luego las dejan actuar por su cuenta". De esta forma, según los autores citados, existen cinco modos de generación del conocimiento:

1. Adquisición: El conocimiento adquirido no tiene por qué haber sido creado recientemente, sólo debe ser nuevo para la organización. La manera más directa y con frecuencia más eficaz de adquirir conocimiento consiste en comprarlo, es decir, comprar una organización o contratar individuos que lo tengan.

2. Asignación de Recursos: Consiste en establecer unidades o grupos específicamente asignados. Los departamentos de investigación y desarrollo son el ejemplo habitual. Sin embargo, debido a que la materialización de los rendimientos de la investigación insume tiempo, puede resultar difícil medirlos cuando se producen.

3. Fusión: Este proceso describe la manera en que la combinación intencional de personas con distintas aptitudes, ideas y valores puede generar soluciones creativas.

4. Adaptación: Esto ocasiona, generalmente, dificultades y los distintos motivos por los que las empresas tienen problemas para adaptarse a los cambios en sus ambientes provienen del hecho fundamental de que la historia es importante, es decir, la capacidad de una empresa para hacer las cosas se desarrolla en el transcurso del tiempo.
5. Redes de Conocimiento: Davenport y Prusak (2001: 76) afirman que "el conocimiento también se genera en redes informales y auto-organizadas en las organizaciones las que posiblemente con el tiempo sean formalizadas". Agregan que los miembros de las comunidades de expertos, reunidos por intereses en común, con frecuencia hablan entre sí, en persona, telefónicamente y mediante correo electrónico para compartir sus conocimientos especializados y resolver los problemas en conjunto.

\section{Dimensión tecnológica de la gerencia del conocimiento}

La aceleración de los cambios tecnológicos, los enfoques competitivos cambiantes en el mercado global y la importancia de la calidad apuntan a la necesidad de una nueva perspectiva para la gerencia. Les corresponde a los gerentes desempeñar un papel crucial en la integración de las acciones competitivas, teniendo en cuenta los conceptos de cadena de valor y percibiendo las oportunidades que se abren con las discontinuidades. Es importante aunar los problemas tecnológicos con el proceso de la gestión de la tecnología, ya que son conceptos que no pueden operar en forma aislada.

Para Ivancevich, Lorenzi y Skinner (2000), las necesidades propias de la tecnología han de gestionarse de la manera más adecuada. Afirman que la calidad es el requisito que da inicio al proceso de gestión de la tecnología. En el marco de referencia sobresalen tres fases distintas: valoración, toma de posición y formación de una política. La fase de valoración indica que la gestión debería efectuar una 
Gerencia del conocimiento en empresas cerveceras

Batlle Rois-Méndez, Francisco y Inciarte Osorio, Bárbara

valoración de la industria y del ambiente, determinar la línea de base (punto de partida) o comienzo de la tecnología actual de la compañía, evaluar las tecnologías de reciente aparición y determinar como la compañía lleva a cabo la transferencia (comercialización) de la tecnología.

Sin embargo, la tecnología está íntimamente relacionada con dos dimensiones clave: la innovación y la creatividad. Para Ivancevich, Lorenzi y Skinner (2000: 713) la innovación puede definirse como "la generación de una nueva idea y su incorporación en un nuevo producto, proceso o servicio". Puede dar lugar al crecimiento económico, a aumentar el empleo y a la generación de beneficios. Es un proceso acumulativo de numerosas decisiones, que abarcan desde la fase de concepción de la idea hasta el desarrollo de tecnología. Por más significativa que pueda ser la inversión tecnológica no puede considerarse como una innovación sino crea crecimiento y beneficio.

Por su parte, Ivancevich, Lorenzi y Skinner (2000: 737) consideran que la creatividad "se define como la capacidad de dar existencia a algo nuevo". Esta generación de algo nuevo puede ser una idea, un proceso e incluso una técnica o estilo. Agregan los autores que produce la sensación de algo misterioso o subversivo.

\section{Dimensión cultural de la gerencia del conocimiento}

Aunque poderosas, las funciones de una solución de la gerencia del conocimiento no tienen una utilidad si no hay voluntad de los participantes. En este sentido, Koulopoulos y Frappaolo (2001) señalan que no se trata simplemente de construir un sistema efectivo, sino de crear una dimensión cultural para compartir conocimiento dentro de la organización, liderizada por un gerente inteligente.

Primero, y ante todo, un gerente inteligente reconoce la necesidad de una comunidad en la organización. Si no existe cualquier intento de propagar el conocimiento es vano. Es importante entender la importancia de crear dentro de la organización un grupo que pueda ayudar a los individuos a encontrar las razones de interés personal que justifique compartir sus conocimientos.

Es común pensar que si el conocimiento existe en la mente de quienes lo poseen, no es susceptible a ser gerenciado, puesto que la gerencia implica control y posesión externos. De esta forma, Koulopoulos y Frappaolo (2001) afirman que un gerente estará rápidamente de acuerdo en que el conocimiento no puede gerenciarse desde la perspectiva de control y posesión externos; pero si se puede gerenciar cuando la meta de la gerencia del conocimiento es fortalecer la capacidad de compartir, y apalancar una base de conocimiento colectivo, y no de tomar posesión de ella.

Al construir una comunidad que comparta conocimiento, se necesita de alguien que quiera compartir conocimiento. Hay organizaciones que tienen la voluntad para compartir conocimiento, pero no hay incentivo para hacerlo. El tema de los incentivos se deriva del hecho de que la persona que busca el conocimiento es quien lo recibe y con él tendrá la capacidad de desempeñar funciones útiles $y$, está lista para recibir ese conocimiento. 
Por su parte, Koulopoulos y Frappaolo (2001) consideran que la medición puede desempeñar un rol significativo para incentivar que se comparta el conocimiento. La medición del conocimiento se refiere a la manera como una organización reconoce que se ha logrado compartir el conocimiento. Hasta que el sistema de medidas con el que la organización cuente esté bien definido, al punto que se pueda comunicar y entender con facilidad en toda la organización, cualquier intento de definir un método puede resultar vano.

\section{Liderazgo en la gerencia del conocimiento}

Como se dijo anteriormente, la gerencia del conocimiento debe ser liderada por un gerente inteligente. Desde esta perspectiva, el liderazgo se concibe como la influencia interpersonal ejercida en determinada situación, para la consecución de uno o más objetivos específicos mediante el proceso de la comunicación humana. Entran en juego dos aspectos: liderazgo e influencia.

Para Chiavenato (2001), el liderazgo es un fenómeno social, un tipo de influjo, que ocurre exclusivamente en grupos sociales. La influencia es una fuerza psicológica que incluye conceptos como poder y autoridad, y se refiere a la manera de introducir cambios en el comportamiento de las personas o grupos de personas. La influencia puede presentarse de diversas maneras, partiendo desde formas violentas de imposición hasta formas suaves de convencimiento. Agrega que el liderazgo es la capacidad de influir en las personas para que ejecuten lo que deben hacer. El líder ejerce influencia en las personas orientando hacia sus objetivos las percepciones de los objetivos de aquéllas. El líder debe ser capaz; los seguidores deben aportar voluntad.

Existe una gran cantidad de opiniones acerca de los diferentes estilos de liderazgo. Una de las más divulgadas es aquella que define tres niveles: liderazgo autocrático, liderazgo liberal y liderazgo democrático. En este sentido, Chiavenato (2001) señala que el líder autocrático es un líder duro e impositivo, el liberal deja que todos hagan su voluntad y el democrático es activo, consultivo y orientador.

La gerencia del conocimiento asocia el liderazgo con dos tendencias específicas: empowerment y coaching. Para Zeus y Skiffington (2002: 101), el empowerment "es un sistema que consiste en potenciar la motivación y los resultados de todos los colaboradores de una empresa a través de la delegación y transmisión de poder".

Por su parte, el coaching es una de las tendencias gerenciales de mayor cabida en la empresa para observar e intervenir los procesos humanos. En este sentido, Zeus y Skiffington (2002: 3) afirman que "el coaching consiste en ayudar a las personas individuales a acceder a lo que saben". Está relacionado con el aprendizaje y con el proceso de formular preguntas apropiadas en lugar de dar las respuestas. 
Gerencia del conocimiento en empresas cerveceras

Batlle Rois-Méndez, Francisco y Inciarte Osorio, Bárbara

\section{Análisis de los hallazgos}

Los hallazgos arrojados al administrar el cuestionario se analizaron considerando la distribución de medias aritméticas (Tabla 1), el marco teórico referencial de la investigación y el contexto organizacional del sector empresarial objeto de estudio.

En relación con el proceso de generación del conocimiento desarrollado en empresas cerveceras, se observó una media aritmética de 4.03, categorizada alta en el baremo, es decir, en las empresas cerveceras se desarrolla altamente la generación del conocimiento atendiendo características bien definidas como la adquisición, la asignación de recursos, la fusión, la adaptación y las redes de conocimiento.

En este sentido, Davenport y Prusak (2001) afirman que cuando se habla sobre generación de conocimiento se hace referencia tanto al conocimiento adquirido por una organización como el desarrollado en la misma. El conocimiento adquirido no tiene por qué haber sido creado recientemente, sólo debe ser nuevo para la organización. Esta es la forma más común como las organizaciones adquieren el conocimiento y partiendo de él se incorporan innovaciones que generan, a la vez, nuevos conocimientos que permiten el logro de las metas y objetivos de la organización.

En la muestra objeto de estudio se verifican procesos de capacitación y adiestramiento que implican la contratación de individuos u organizaciones que se encargan de diseñar experiencias de aprendizaje para los empleados de la organización. Asimismo, la adquisición de empresas incluye el conocimiento generado por esas empresas. Los autores $\mathrm{Da}$ venport y Prusak (2001: 68) afirman que

Tabla 1

Distribución de medias aritméticas de los indicadores y dimensiones de la variable gerencia del conocimiento

\begin{tabular}{|c|c|c|c|c|c|c|c|c|c|c|c|c|}
\hline \multirow{3}{*}{$\begin{array}{l}\text { Variable } \\
\text { Dimensiones } \\
\text { Indicadores }\end{array}$} & \multicolumn{12}{|c|}{ Gerencia del Conocimiento } \\
\hline & \multicolumn{4}{|c|}{$\begin{array}{c}\text { Generación } \\
\text { del Conocimiento }\end{array}$} & \multicolumn{3}{|c|}{$\begin{array}{l}\text { Dimensión } \\
\text { Tecnológica }\end{array}$} & \multicolumn{4}{|c|}{ Dimensión Cultural } & Liderazgo \\
\hline & Adq. & As. Rec. & Fus. & Ada. & $\begin{array}{l}\text { Red. } \\
\text { Cto. }\end{array}$ & Inn. & Cre. & $\mathrm{Cm}$. & Pr. & Inc. & Me. & Tip. \\
\hline $\mathbf{X}_{\text {INDICADOR }}$ & 4.47 & 4.37 & 3.73 & 4.26 & 3.32 & 4.45 & 4.53 & 3.95 & 3.87 & 3.84 & 3.34 & 4.11 \\
\hline Categoría & M.A. & M.A. & Alta & M.A. & Med. & M.A. & M.A. & Alta & Alta & Alta & Med. & Alta \\
\hline$X_{\text {DIMENSIÓN }}$ & & & 4.03 & & & & & & 3.75 & & & 3.98 \\
\hline Categoría & & & Alta & & & Muy & Alta & & Alta & & & Alta \\
\hline $\mathbf{X}_{\text {VARIABLE }}$ & & & & & & 4.0 & & & & & & \\
\hline Categoría & & & & & & Alt & & & & & & \\
\hline
\end{tabular}

Fuente: Batlle e Inciarte (2005). 
"centrarse en los beneficios a corto plazo puede crear presión para reducir los costos mediante la supresión de la investigación y el desarrollo".

El resultado de la combinación intencional de personas con distintas aptitudes, ideas y valores puede generar soluciones creativas. Las empresas cerveceras que creen firmemente en el potencial creativo de la fusión adoptan decisiones de contratación, específicamente, para promover la diversidad cognoscitiva en la empresa y une, por ejemplo, un nuevo empleado analítico y racional con uno con inclinaciones intuitivas y estéticas.

Cabe destacar que este resultado representa una ventaja competitiva para la población consultada pues no se limita a asimilar conocimientos desarrollados en otras organizaciones sino que, por el contrario, construye aprendizajes y procesos fundamentados en sus necesidades organizacionales, financieras y administrativas.

En relación con la dimensión tecnológica con la cual se administra la gerencia del conocimiento en empresas cerveceras, se observó una media aritmética de 4.49 ubicada en la categoría muy alta del baremo establecido, considerando que los aspectos tecnológicos medidos, innovación y creatividad, se administran muy altamente al momento de gerenciar el conocimiento de la organización.

Para Koulopoulos y Frappaolo (2001: 5), una organización que gerencia el conocimiento "asume una vigilancia constante y estimula la modificación e innovación permanentes, a una velocidad que, por lo menos, le sigue el paso a la dinámica del mercado cambiante". El gerente del conocimiento reconoce que en el mercado actual el cambio es inevitable $y$, por consiguiente, la innovación debe ser constante. Cabe destacar que para seguir siendo competitivo, se debe cosechar el conocimiento del pasado y construir sobre él para responder al mercado del mañana.

También es importante el razonamiento de Ivancevich, Lorenzi y Skinner (2000: 737), al señalar que la creatividad "se define como la capacidad de dar existencia a algo nuevo". En consecuencia, la creatividad es parte integrante de los aspectos tecnológicos de la gerencia del conocimiento por cuanto, a través de ella, se van generando nuevos conocimientos susceptibles a ser gerenciados dentro de la organización. En este sentido, se observó sincronía entre los resultados observados en los sujetos y lo establecido teóricamente por Koulopoulos y Frappaolo (2001) e Ivancevich, Lorenzi y Skinner (2000).

En relación con la dimensión cultural desarrollada por la gerencia del conocimiento en empresas cerveceras, se observó una media aritmética de 3.75 , ubicando esta dimensión en la categoría alta del baremo, es decir, en las empresas cerveceras se toman en cuenta altamente, los aspectos culturales al momento de gerenciar el conocimiento, específicamente la construcción de comunidades de conocimiento, la propiedad del conocimiento, los incentivos y las mediciones.

En este sentido, Koulopoulos y Frappaolo (2001) plantean que un gerente inteligente estará rápidamente de acuerdo en que el conocimiento no puede gerenciarse; pero que, a la vez, se sabe que la meta de la gerencia del conocimiento es fortalecer la capacidad de com- 
Gerencia del conocimiento en empresas cerveceras

Batlle Rois-Méndez, Francisco y Inciarte Osorio, Bárbara

partir, y apalancar una base de conocimiento colectivo, y no de tomar posesión de ella.

De esta forma se confirman sus planteamientos cuando afirman que al construir una comunidad que comparta conocimiento, se necesita de alguien que quiera compartir conocimiento. Hay organizaciones que tienen la voluntad para compartir conocimiento, pero no hay incentivos para hacerlo. Como se observó, fue detectada concordancia entre los resultados observados en los sujetos y lo establecido teóricamente por Koulopoulos y Frappaolo (2001).

En relación con el liderazgo ejercido por los gerentes el conocimiento en empresas cerveceras, se observó una media aritmética de 3.98 categorizada alta en el baremo establecido, es decir, que se desarrolla altamente un liderazgo democrático y participativo que se apoya en tendencias gerenciales actuales como el empowerment y el coaching.

Estos resultados coinciden con Chiavenato (2001) cuando señala que el liderazgo es la influencia interpersonal ejercida en determinada situación, para la consecución de uno o más objetivos específicos mediante el proceso de la comunicación humana. La influencia puede presentarse de diversas formas tal como lo indican los planteamientos de Zeus y Skiffington (2002) cuando afirman que el empowerment es la herramienta mediante la cual los individuos, a nivel individual o como miembros de una organización, se sienten competentes, considerándose eficaces y sintiéndose integrados en la empresa, en la que asumen el nivel más alto de delegación por parte de sus superiores.
Al mismo tiempo, los resultados coinciden con las afirmaciones de Zeus y Skiffington (2002) al señalar que un coach es un profesional que se vale de la sistematización y diseño de aprendizajes, como herramientas de trabajo dirigidas a resolver dificultades recurrentes de relación ante las cuales se encuentran un individuo, grupo u organización, intensificando la observación profunda de sí mismo y la circunstancia que la afectan.

Estos hallazgos enmarcan la gestión de las empresas cerveceras dentro de la teoría de las empresas que aprenden, postulada por Garvin (2000), donde se hace énfasis en el intento de las empresas por perfeccionarse y obtener ventajas competitivas, señalando en este aspecto la causa de la proliferación de los programas de mejora continua.

\section{Conclusiones}

1. Se caracterizó el proceso de generación de conocimiento desarrollado en empresas cerveceras destacándose significativamente la adquisición de conocimiento, la asignación de recursos, la fusión y la adaptación. Sin embargo, se verificó que la población objeto de estudio presenta debilidades en relación con la formación de redes de conocimiento como una forma pertinente para generar conocimiento sobre la base del ya existente y como estrategia para ampliar el radio de acción de los conocimientos generados.

2. Se analizaron la innovación y la creatividad como las dimensiones tecnológicas con las cuales las empresas cerveceras administran el conocimiento como estrategia para posicionarse en el 
mercado. Dentro de los valores organizacionales de la empresa, la innovación y la creatividad se conjugan para generar procesos que le permitan mantenerse como empresa líder en el ramo. Es a través de la innovación y la creatividad como genera conocimientos que fundamentan la tecnología de punta que respalda la elaboración de los productos que ofrecen al consumidor.

3. Se establecieron como dimensiones culturales que desarrolla la gerencia del conocimiento en empresas cerveceras: la construcción de comunidades, la propiedad del conocimiento, los incentivos y las mediciones. Entre ellas se destaca la construcción de comunidades como el indicador más significativo por cuanto el trabajo en equipo es otro de los valores que las empresas potencian en sus trabajadores, de forma tal que el conocimiento es compartido entre los miembros de los equipos de trabajo.

Este hecho pareciese que contrastara con la debilidad que se mencionó en la primera conclusión, en relación con las redes de conocimiento. Sin embargo, se verificó que se construyen comunidades que comparten sus aprendizajes pero, a la vez, son cerradas para compartirlos con otros equipos con los que pudiesen formar una red.

4. Se estableció un liderazgo transformador ejercido por los gerentes del conocimiento de empresas cerveceras. Se evidenció que el liderazgo está dirigido a fomentar el trabajo en equipo como valor organizacional de la población objeto de estudio, fundamentado en las tendencias gerenciales del empowerment y el coaching, de modo que se delegan mayores responsabilidades a los trabajadores $y, a$ la vez, se les potencia y se les prepara para que asuman los retos personales, profesionales y laborales.

5. Se determinó que la generación del conocimiento, la dimensión tecnológica, la dimensión cultural y el liderazgo son los principales elementos de la gerencia del conocimiento desarrollada en empresas cerveceras; destacándose el desarrollo de los aspectos tecnológicos como la estrategia que le ha permitido a la población objeto de estudio mantenerse como líder en el mercado gracias a las innovaciones y la tecnología de punta que aplica a los procesos productivos.

\section{Referencias Bibliográficas}

Argyris, Chris (2000). Como Dirigir Personas en las Empresas. Harvard Business Review. Bilbao: Deusto.

Chiavenato, Idalverto (2001). Administración. México: Mc Graw-Hill.

Davenport, Thomas y Prusak, Laurence (2001). Conocimiento en Acción. Buenos Aires: Prentice Hall.

Ferrer, José (2001). La Gerencia del Conocimiento y la Educación. Maracaibo, 04 de julio: Diario Panorama.

Garvin, David (2000). Crear una Organización que Aprende. Harvard Business Review. Bilbao: Deusto.

Harvard Business Review (2000). Gestión del Conocimiento. Bilbao: Deusto.

Hernández, Roberto; Fernández, Carlos; Baptista, Pilar (2006). Metodología de la Investigación. México: Mc. Graw Hill.

Honeycutt, Jerry (2001). Así es la gestión del Conocimiento. Madrid: Mc Graw-Hill Interamericana.

Ivancevich, John; Lorenzi, Peter; Skinner, Steven (2000). Gestión, Calidad y Competitividad. Madrid: Mc Graw-Hill. 
Gerencia del conocimiento en empresas cerveceras

Batlle Rois-Méndez, Francisco y Inciarte Osorio, Bárbara

Koulopoulos, Thomas y Frappaolo, Carl (2001). Lo Fundamental y lo más Efectivo acerca de la Gerencia del Conocimiento. Bogota Colombia: Mc Graw-Hill.

Nonaka, Ikujiro (2000). La Empresa Generadora de Conocimiento. Harvard Business Review. Bilbao: Deusto.

Pavez, Alejandro (2000). La Gestión del Conocimiento en las Organizaciones [Documento en línea]. Disponible: http://www.gestiondelconocimiento.com [Consulta: 2004, Julio 03].

Udaondo, Miguel (2000). La Gestión del Conocimiento [Documento en línea]. Disponible: http://www.gestiondelconocimiento.com [Consulta: 2004, Julio 03].

Zeus, Perry y Skiffington, Suzanne. (2002). Guía Completa de Coaching en el Trabajo. Madrid: Mc Graw Hill. 